\title{
Factor Saving Innovation
}

\author{
Michele Boldrin and David K. Levine \\ University of Minnesota and UCLA
}

July 18, 2001

\begin{abstract}
It has been argued that concave models exhibit less "endogeneity of growth" than models with increasing returns to scale. Here we study a simple model of factor saving technological improvement in a concave framework. Capital can be used either to reproduce itself, or, at some additional cost, to produce a higher quality of capital, which requires less labor input. If better quality capital can be produced quickly, we get a model of exogenous balanced growth as a special case of ours. If, however, better quality capital can be produced slowly, we get a model of "endogenous growth" in which the growth rate of the economy and the rate of adoption of new technologies is determined by preferences, technology and initial conditions. Moreover, in the latter case, the process of growth is necessarily uneven, exhibiting a natural cycle with alternating periods of high and slow growth. Growth paths and technological innovations also exhibit dependence upon initial conditions. The model provides a step toward a theory of endogenous innovation under conditions of perfect competition.
\end{abstract}




\section{Introduction}

We contribute to the debate on the endogeneity of aggregate technological progress by introducing a concave model of innovation with three properties. Technological innovations are: (a) factor saving; (b) implementable only in discrete lumps; (c) endogenous, depending on people's decisions. We find in such circumstances that growth can be path-dependent and uneven over time.

In our model, technological progress takes place through the adoption of new activities that make use of new qualities of capital. There is a fixed factor, and more advanced activities are superior in the sense that they make less use of this fixed factor. This means that technological progress is "biased" or "factor saving." For concreteness, we refer to the fixed factor as labor. In this setting investment provokes both capital widening, meaning the total stock of capital grows larger, and capital deepening, meaning that the quality of the capital stock is improved. In fact, because of the fixed labor supply, capital deepening is necessary for capital widening.

Without moving to higher qualities of capital that use less labor per unit of output, there is no reason to build more capital. Introducing new capital goods is costly because, for given inputs, the capital deepening technology yields less units of output than the capital widening technology. Hence regardless of how long the capital deepening activity has been available, it will be used only when relative prices make it profitable to do so; that is, when the labor supply becomes a binding constraint. In this sense, technological progress is fully endogenous.

Our model is one of perfect competition with constant returns to scale. A variety of arguments have been advanced as to why growth models with increasing returns are superior to those with diminishing or constant returns. From a theoretical standpoint, the endogenous versus exogenous nature of economic growth is the principal one. Romer [1994], for example, says that the fact that "technical advance comes from things that people do" and is not merely "a function of elapsed calendar time," argues against concave models of "exogenous" technological change. In this interpretation, endogeneity means that technological innovations should come from "things people do".

In our setting technical advance clearly comes about because it is profitable for innovators to introduce new technologies into production. This should clarify that growth can be as endogenous in a concave setting as it is when there are externalities, increasing returns, monopoly power and so forth. To the best of our knowledge, this is the first time such a result is clearly proven. 
Our equilibrium path distinguishes between growth due to the accumulation of factors, and the introduction of new factors and activities, which we refer to as technological advance. To be concrete, we will propose that the growth rate or the rate of technological advance are endogenous if they are affected in a non-trivial way by changes in the rate of intertemporal substitution in consumption. Notice that in the Solow growth model, neither the growth rate nor the rate of technological advance are endogenous in this sense. In Jones and Manuelli's [1990] and Rebelo's [1991] AK model, the growth rate is endogenous, but the rate of technological advance is not. On the other hand, in models with increasing returns such as those of Lucas [1988] or Romer [1990], not only is the growth rate endogenous, but so is the rate of technological advance.

The endogeneity of growth in this model depends on how rapidly it is possible to produce higher quality capital. If capital of higher quality can be produced quickly, we get a model identical in essence to the "exogenous growth" model of Solow: A new quality of capital is introduced every period, and the economy grows at a fixed rate independent of the subjective discount factor and other preference or technology parameters. If new quality capital can only be produced slowly, the situation changes drastically. Both the growth rate and the rate of technological advance are fully endogenous and depend on the subjective discount factor and other parameters of preferences.

In this model, technical advances clearly come from things that people do. In fact, contrary to models where externalities carry the day, technological improvements here come from things that people consciously choose to do. They introduce new technologies in those periods when they are needed to relax the labor constraint, and they do not introduce new technologies in periods in which such need is absent. Note that we do not attempt to model the reason that technologies become available. We assume that technologies are available or become available for reasons exogenous to the model. Our theory of technological progress is a theory by which those technologies are actually introduced into use. A theory of why new technologies become available in a concave world is presented in Boldrin and Levine [2000].

As we mentioned at the outset, the most striking features of this model is that equilibria are path dependent and do not exhibit a constant growth rate. Growth follows a natural cycle in which gradual upward increases in consumption are interrupted by periodic "growth recessions" in which consumption remains flat. These periods of "creative destruction" are those in which a shift to a new technological paradigm first takes place. The existence of growth cycles can be extended to models with many goods, sectors and factors of production, as long as natu- 
ral resources are essential in production and innovation has an impact on many sectors. While it can be argued that many innovations are cumulative in nature, so that the introduction of a new technology has only a slight effect on the economy, innumerable important innovations such as the use of personal computers, the introduction of electrical power, or the advent of new business methods, are of general purpose and can be expected to have a substantial impact across many sectors. In the presence of a sequence of such "large" innovations, the process of growth will be uneven with spurts of growth as the new technology is exploited, and periods of relative stagnation while the new capital good is accumulated for the next growth "spurt." Note that during growth recessions the economy remains at full employment; unemployment occurs only in the case of stagnation when the capital stock becomes too small to employ all existing labor.

In addition to endogenous growth and a natural growth cycle, our economy exhibits path dependence meaning that the long run growth rate of the economy can depend on the initial stock of capital. Indeed, a small change in initial capital can make the difference between long-run innovation and growth, and long-run stagnation and decline. In particular, it is possible for the economy to grow in the short-run, introducing new technologies and increasing consumption per capita, yet in the long-run fall back into stagnation with declining consumption, unemployment and only the worst possible technology employed. Again, and to the best of our knowledge, we are not aware of any dynamic model exhibiting this rather frequently observed pattern.

\section{The Model}

We consider an infinite horizon economy, $t=0,1,2, \ldots$ with a continuum of homogeneous consumers. Consumers value consumption $c_{t} \in \mathfrak{R}_{+}$. The period utility function $u\left(c_{t}\right)$ is bounded below, continuously differentiable, strictly increasing, and strictly concave. It satisfies the Inada conditions $\lim _{c \rightarrow 0} u^{\prime}(c)=+\infty$ and $\lim _{c \rightarrow+\infty} u^{\prime}(c)=0$. Total lifetime utility is given by $U(c)=\sum_{t=0}^{\infty} \delta^{t} u\left(c_{t}\right)$, where $0 \leq \delta<1$ is the common subjective discount factor. Let $\mu=\sup \left\{\widetilde{\mu} \| \sum_{t=0}^{\infty} \delta^{t} u\left(\widetilde{\mu}^{t}\right)<\right.$ $\infty$ to be the supremum of growth rates for which utility remains finite. Notice that $\mu \geq 1 / \delta$.

Consumption is produced by activities that use labor and capital as inputs. In addition, capital is produced from capital, and labor reproduces itself. Capital comes in an infinite sequence of different qualities, indexed by $i=0,1, \ldots$. 
Write an input vector as $z=(\kappa, \ell)$ where $\kappa$ is an infinite vector of capital stocks of different qualities, and $\ell$ is a scalar denoting labor. The period input space $Z$ consists of the set of sequences $\left(z_{1}, z_{2}, \ldots, z_{n}, \ldots\right) \geq 0$ for which $z_{n}=0$ for all but finitely many $n$. Note that the technology and initial condition are such that in any particular period it is not possible to have produced more than a finite number of different qualities of capital, so there is no loss of generality in this restriction. We let the symbol $\chi_{i}$ denote the vector consisting of one unit of capital $i$ and zero units of all other capitals. So, for example, $\left(\chi_{2}, 0\right)$ is an input vector with 1 unit of quality 2 capital and zero units of everything else. The period commodity space is $Z \times \Re_{+}$.

The set of all possible activities $a$ is denoted by $\mathcal{A}$. An activity $a \in \mathcal{A}$ may be written as a vector consisting of a triplet $\left[z(a) ; z^{+}(a) ; c(a)\right]$, where $z(a), z^{+}(a) \in$ $Z$ and $c(a) \in \mathfrak{R}_{+}$. Here $z(a)=[\kappa(a), \ell(a)]$ represents the input requirement for activity $a$ in period $t, z^{+}(a)=\left[\kappa^{+}(a), \ell^{+}(a)\right]$ represents the output of period $t+$ 1 inputs produced by activity $a$, and $c(a)$ represents consumption produced by activity $a$ and available in period $t$.

Our basic assumption is that capital of quality $i$ can be used to produce consumption, capital of the same quality or capital of the next higher quality. We assume that labor is an input (and also an output) in the production of consumption, but not in the production of capital. While this is just a simplifying assumption, as we discuss in the conclusion, it is consistent with the idea that there is little labor mobility between sectors. In any case, as we discuss in the conclusion, even if we were to allow labor mobility, the general nature of our results do not change.

Specifically, there is a sequence of activities for producing consumption, one for each type of capital $i$. For quality $i$ capital, the activity is $\left[\chi_{i}, 1 / \gamma^{i} ; 0,1 / \gamma^{i} ; 1\right]$, $\gamma>1$. In other words, to produce a unit of consumption requires a unit of capital (of any quality) and a number of units of labor that is smaller the higher is the quality of the capital. The assumption $\gamma>1$ embodies the notion that technological progress is labor saving. Notice that labor appears here both as an input and as an output, so that labor that is used in production today remains available for production tomorrow.

Two sequences of activities can produce capital. They are $\left[\chi_{i}, 0 ; \beta \chi_{i}, 0 ; 0\right], \beta>$ 1 and $\left[\chi_{i}, 0 ; \rho \chi_{i+1}, 0 ; 0\right], \rho>0$. This means that the current quality of capital may be used either to produce $\beta$ units of the same quality of capital (capital widening) or $\rho$ units of the next quality of capital (capital deepening). We set $\beta>\rho$, so that introducing the next quality of capital goods instead of widening the current one is costly because it requires a sacrifice of current consumption. We assume that $\mu>\min \{\beta, \gamma\}$ so maximum utility over feasible consumption paths is finite. 
We also assume that there is free disposal, and an activity that produces next period labor by means of current period labor $[0,1 ; 0,1 ; 0]$. In conjunction with the assumption that labor is an output of the activity that produces consumption, it guarantees that labor reproduces itself in each period, hence it is always available in a constant amount. One can make labor supply grow at some rate $n<\beta-1$ by modifying the output vectors of these two activities appropriately. Results would not change.

The endowment $z_{0}=\left(\kappa_{0}^{0} \chi_{0}, 1\right)$ consists of $\kappa_{0}^{0}$ units of quality 0 capital and one unit of labor.

\subsection{Equilibrium}

A $\lambda \in\left(\times_{t=0}^{\infty} \Re_{+}^{\mathcal{A}}\right)$ is called a production plan, a $c \in\left(\times_{t=0}^{\infty} \Re_{+}\right)$is called a consumption plan. Together they determine an (intertemporal) allocation.

Definition 1. The allocation $\lambda, c$ is a feasible allocation for the initial condition $z_{0}$ iffor all $t \geq 0$

$$
\begin{aligned}
1 & \geq \sum_{a \in \mathcal{A}} \lambda_{0}(a) \ell(a) \\
\kappa_{0}^{0} \chi_{0} & \geq \sum_{a \in \mathcal{A}} \lambda_{0}(a) \kappa(a) \\
\sum_{a \in \mathcal{A}} \lambda_{t}(a) z^{+}(a) & \geq \sum_{a \in \mathcal{A}} \lambda_{t+1}(a) z(a)
\end{aligned}
$$

Definition 2. The allocation $\lambda^{*}, c^{*}$ solves the social planner problem for initial condition $z_{0}$ if it solves

$$
\max _{\lambda, c} U(c)
$$

subject to feasibility of the allocation.

Notice that in a feasible production plan $\lambda_{t}(a)=0$ if $a$ uses as input any quality of capital greater than $t$. Denote by $A_{t}$ the set of viable activities which use as input qualities of capital no greater than $t$.

Let $q_{t}^{i}$ denote the price of quality $i$ capital at time $t$, let $q_{t}^{\ell}$ denote the price of labor at time $t$, and let $p_{t}$ denote the price of consumption at time $t$. We denote by $q_{t}$ the vector of all input prices at time $t$, and let $q$ and $p$ denote, respectively, the infinite sequence of prices of the two inputs and consumption starting in period 
0 . Prices $q, p$ and a feasible allocation $\lambda, c$ are a competitive equilibrium if $c$ maximizes $U(c)$ subject to the budget constraint

$$
\sum_{t=0}^{\infty} p_{t} c_{t} \leq q_{0}^{0} \kappa_{0}^{0}+q_{0}^{\ell}
$$

and activities satisfy the zero profit condition

$$
q_{t+1} \cdot\left[\kappa^{+}(a), \ell^{+}(a)\right]+p_{t} c(a)-q_{t} \cdot[\kappa(a), \ell(a)] \leq 0, \forall a \in A_{t}, t=0,1, \ldots
$$

with equality if $\lambda_{t}(a)>0$.

In the appendix we prove the relevant version of the First and Second Welfare Theorems:

Theorem 1. Suppose that $\lambda^{*}, c^{*}$ is a feasible allocation for the initial condition $z_{0}$. Then $\lambda^{*}, c^{*}$ solves the social planner problem if and only if we can find prices $q, p$ such that $q, p, \lambda^{*}, c^{*}$ are a competitive equilibrium.

The following existence and uniqueness result is also proved in the appendix.

Theorem 2. For given $z_{0}$, a competitive equilibrium exists, and there is a unique competitive equilibrium consumption plan $c^{*}$.

We can now use the first order conditions to give a relatively simple characterization of equilibrium consumption paths. First we begin by calculating the least amount of initial capital needed to produce a given consumption in a particular period. Given a particular value of $c_{t}$ observe that either $c_{t} \leq 1$ or, for some $i>0$, $\gamma^{i-1}<c_{t} \leq \gamma^{i}$. In the former case define $\eta\left(c_{t}\right)=0$, in the latter $\eta\left(c_{t}\right)=i$. In this way $\eta(c)$ indexes the highest quality of capital needed to afford a per-capita consumption level equal to $c$. Define

$$
\kappa_{0}^{0}\left(c_{t}\right)=\left\{\begin{array}{cc}
\beta^{-t} c_{t} & \eta\left(c_{t}\right)=0 \\
\beta^{-t}\left(\frac{\beta}{\rho}\right)^{\eta\left(c_{t}\right)} \frac{\gamma c_{t}-\gamma^{\eta\left(c_{t}\right)}}{\gamma-1}+\beta^{-t}\left(\frac{\beta}{\rho}\right)^{\eta\left(c_{t}\right)-1} \frac{\gamma^{\eta\left(c_{t}\right)}-c_{t}}{\gamma-1} & \eta\left(c_{t}\right)>0 .
\end{array}\right.
$$

The latter expression represents "the amount of initial capital required to produce $c_{t}$ when it is produced using only qualities $\eta\left(c_{t}\right)$ and $\eta\left(c_{t}\right)-1$ capital". Using this we define also the "initial capital requirement to produce the consumption plan $c$ "

$$
\kappa_{0}^{0}(c)=\sum_{t=0}^{\infty} \kappa_{0}^{0}\left(c_{t}\right)
$$


Next we define a correspondence that captures the first order conditions for an optimal path. Our basic strategy is to find an optimal path for a given price of initial capital $q_{0}^{0}$, then back out the initial condition. First we define the constants

$$
\begin{aligned}
& \zeta_{0}=1 \\
& \zeta_{i}=(\beta / \rho)^{i-1}[(\beta \gamma / \rho)-1] /(\gamma-1) .
\end{aligned}
$$

For $q_{0}^{0} \geq 0$ and $t=0,1, \ldots$, we use these constants to define the a correspondence $c_{t}^{\prime} \in C_{t}\left(c_{t}, q_{0}^{0}\right)$ from $c_{t} \in\left[0, \gamma^{t}\right]$ into $\Re_{+}$

$$
\begin{array}{ll}
u^{\prime}\left(c_{t}^{\prime}\right)=(\beta \delta)^{-t} q_{0}^{0} \zeta_{\eta\left(c_{t}\right)} & \text { if } c_{t}<\gamma^{\eta\left(c_{t}\right)}, \eta\left(c_{t}\right) \leq t \\
(\beta \delta)^{-t} q_{0}^{0} \zeta_{\eta\left(c_{t}\right)} \leq u^{\prime}\left(c_{t}^{\prime}\right) \leq(\beta \delta)^{-t} q_{0}^{0} \zeta_{\eta\left(c_{t}\right)+1} & \text { if } c_{t}=\gamma^{\eta\left(c_{t}\right)}, \eta\left(c_{t}\right)<t \\
(\beta \delta)^{-t} q_{0}^{0} \zeta_{\eta\left(c_{t}\right)} \leq u^{\prime}\left(c_{t}^{\prime}\right) & \text { if } c_{t}=\gamma^{\eta\left(c_{t}\right)}, \eta\left(c_{t}\right)=t
\end{array}
$$

This correspondence consists of horizontal and vertical line segments forming the steps of a "descending" stair as shown in Figure 1.

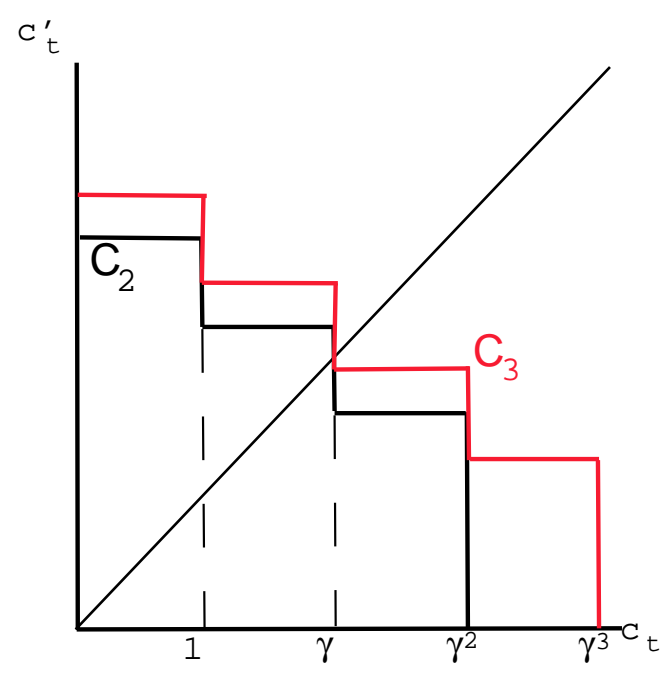

Figure 1 - Stairstep Correspondence

Because of its stairstep nature, the correspondence is upper-hemi-continuous, convex valued and non-increasing. It is immediate to see that, for given $q_{0}^{0}$ and $t$, it has only one fixed point $c_{t}^{*} \in\left(0, \gamma^{t}\right]$. Notice that the location of the stairsteps is always at $\gamma^{i}, i=0,1, \ldots$. The stair is always truncated at $\gamma^{t}$ since no type of capital greater than $i=t$ can be used. For this reason as $t$ increases the end of the stairstep always moves to the right. Whether the vertices move up or down with 
$t$ depends on $\beta \delta$. Notice that $u^{\prime}(\cdot)$ is a decreasing function and $\beta \delta$ is raised to a negative power of $t$. So, if $\beta \delta>1$ the stairs get higher; if $\beta \delta<1$ the stairs get lower. The figure shows $C_{2}, C_{3}$ in the case in which $\beta \delta>1$. We will exploit these basic observations below in analyzing equilibrium consumption paths.

The idea behind the $C$ correspondence is to consider that $c_{t}$ determines the price of consumption through $\delta^{t} u^{\prime}\left(c_{t}\right)$. Given the price of consumption and the initial price of capital $q_{0}^{0}$ we can calculate in period $t$ how profitable it is to use each different viable quality of capital to produce consumption. Inspection of the profit conditions show that they are concave as a function of the index $i$. This means that at most two activities can yield zero profits when all others yield nonnegative profits. We then plug the prices $\delta^{t} u^{\prime}\left(c_{t}\right), q_{0}^{0}$ into the two activities that earn zero profits. Observe that using these two (or one) activities together with full employment yields exactly $c_{t}^{\prime}$ units of consumption. Consequently, the correspondence is defined by the condition that the amount of consumption giving rise to the prices should equal the amount of consumption generated from the production technology when the zero profit conditions are satisfied. In other words, the key property of the correspondence $C$ is that its fixed points capture the first order conditions for an optimal path. In the appendix we formally prove:

Theorem 3. For given $z_{0}$ the feasible consumption plan $c^{*}$ is an optimum if and only if there exists a $q_{0}^{0}$ such that

$$
\begin{gathered}
\kappa_{0}^{0} \geq \kappa_{0}^{0}\left(c^{*}\right) \text { with equality unless } q_{0}^{0}=0 \\
\qquad c_{t}^{*} \in C_{t}\left(c_{t}^{*}, q_{0}^{0}\right)
\end{gathered}
$$

Moreover, equilibrium prices are given by the following

$$
\begin{gathered}
q_{t}^{i}=\beta^{-t}\left(\frac{\beta}{\rho}\right)^{i} q_{0}^{0}, \\
w_{t}=\gamma^{\eta\left(c_{t}^{*}\right)}\left[\delta^{t} u^{\prime}\left(c_{t}^{*}\right)-\beta^{t}(\beta / \rho)^{\eta_{t}} q_{0}^{0}\right]
\end{gathered}
$$

and

$$
q_{T}^{\ell}=\sum_{t=T}^{\infty} w_{t}
$$

The equilibrium production plan is any feasible plan that produces $c_{t}^{*}$ using only quality $\eta\left(c_{t}^{*}\right)$ and $\eta\left(c_{t}^{*}\right)-1$ quality capital, and has full employment whenever $\eta\left(c_{t}^{*}\right)>0$. 


\section{Solow, Growth Cycles and Stagnation}

Here we focus on the long-run behavior of the economy. We show that, depending on parametric configurations and initial conditions there are three possible longrun outcomes.

In the first, a new quality of capital is introduced every period and the economy grows at the rate $\gamma$, independently from preferences and other technological parameters. We refer to this as the Solow growth path. For the technology which is being considered here, the Solow path provides the highest attainable level of consumption in each single period. At the opposite extreme, only the poorest quality of capital is used and the capital stock either declines or remains the same forever. We refer to this second outcome as the stagnation steady state. While possible in principle, both the Solow growth path and stagnation are very unlikely outcomes requiring extreme configurations of the parameter values. Finally, the economy may enter an irregular growth cycle, in which two different qualities of capital are used for a period of time, then the lower quality capital is dropped and a new quality of capital is introduced for the first time, and so on. We refer to this as the growth cycle. This is the main focus of our interest.

We first study the Solow balanced growth path, which is the easiest and next the growth cycle, which is the most interesting. We conclude with the special case of stagnation.

\subsection{The Solow Balanced Growth Path}

Improving the quality of capital does not change the amount of output that can be produced from that capital, but it does reduce the labor requirement for one unit of output. Since there is a fixed supply of labor, the economy can grow, but only by continually moving to more advanced qualities of capital that make it possible to produce increased amounts of output from the existing stock of labor. When the innovation occurs, $\rho$ units of new capital are produced for each unit of old capital invested, generating an additional demand of $\rho / \gamma-1$ units of labor input. If $\rho>\gamma$ the latter quantity is positive and, in each period, it is possible to shift the entire stock of capital from one quality to the next without causing labor to be unemployed. If, at a certain point in time, there is enough capital to employ all available labor and, from that period onward, a new quality of capital is introduced each period, the capital stock can grow fast enough that all labor remains employed on capital of the newest quality. In this case the rate of technological progress is independent of preferences and, also independent of preferences, con- 
sumption grows at the fixed rate $\gamma$. We refer to this as the Solow growth path, since this is the same result as in the Solow growth model with exogenous technological progress.

If $\rho>\gamma$ and the initial capital stock is large enough, then the unique equilibrium is this Solow growth path beginning with consuming a unit in period one. Notice that if this path is feasible it must be optimal, since it is not possible by any plan to have higher consumption in any period.

Recall that $\kappa_{0}^{0}$ is the initial stock and let $\kappa_{t}^{i}$ denotes the capital stock of quality $i$ at time $t$. Along a Solow growth path, at $t$ only $\kappa_{t}^{t}$ is positive. Suppose that $\kappa_{0}^{0}$ is larger or equal to the least capital stock needed for the Solow growth path to be feasible. Then we must have $\kappa_{1}^{1}=\gamma \kappa_{0}^{0}$. In addition a unit of capital must be used to produce consumption of 1 in period 0 , so $\kappa_{1}^{1}=\rho\left(\kappa_{0}^{0}-1\right)$. Solving, we find that an initial stock of capital equal, at least, to

$$
\kappa_{0}^{0}=\frac{\rho}{\rho-\gamma}
$$

is needed to make the Solow path feasible. We summarize by

Theorem 4. If $\rho>\gamma$ and $\kappa_{0}^{0} \geq \rho /(\rho-\gamma)$, the unique equilibrium is a balanced growth path in which a new technology is introduced every period, consumption in period $t$ is $\gamma^{t}$, capital also grows at the rate $\gamma$ and there is full employment in all periods.

Next we look at the behavior of prices, factor shares and observable TFP along the Solow path. Notice first that along a Solow path $\eta_{t}=t$ all $t$. Further, for $c^{*}=\left\{\gamma^{t}\right\}_{t=0}^{\infty}$ the initially required capital stock is $\kappa_{0}^{0}\left(c^{*}\right)=\frac{\rho}{\rho-\gamma}$. Hence we can take $q_{0}^{0}=0$ if $\kappa_{0}^{0}>\frac{\rho}{\rho-\gamma}$. In fact, in this Solow case, we may take the initial price of capital $q_{0}^{0}=0$, also for $\kappa_{0}^{0}=\frac{\rho}{\rho-\gamma}$ since utility does not increase with increases in the capital stock. This implies also that the prices of all qualities of capital in all periods is zero. Normalizing the marginal utility of income $\psi=1$, the consumption prices are

$$
p_{t}=\delta^{t} u^{\prime}\left(\gamma^{t}\right)
$$

Wages are

$$
w_{t}=\gamma^{t} p_{t}
$$

and the real wage $\tilde{w}_{t}=w_{t} / p_{t}=\gamma^{t}$, so real wages grow exponentially over time.

Notice that, independently from our normalization of the initial price of capital, output grows at a constant and exogenous rate $\gamma$ and factor shares are constant 
at the level determined in the first period. The capital/labor ratio is also growing at the constant rate $\gamma$. Similarly for "effective" or, as we call it here, enhanced labor with the productivity of physical labor growing at the exogenous rate $\gamma$. Hence our golden age is observationally equivalent to the traditional Solow growth model, with a Cobb-Douglas production function and exogenous technological progress.

\subsection{The Growth Cycle}

When circumstances are not so lucky, that is when either $\gamma<\rho$ or the initial stock of capital $\kappa_{0}^{0}<\rho /(\rho-\gamma)$ is too low to make the Solow path immediately accessible, the long run behavior of both consumption and the introduction of new technologies will generally depend upon preferences, and in particular on the subjective discount factor $\delta$. There are two cases, depending on whether $\delta \beta>1$, or $\delta \beta \leq 1$. If there was no labor constraint and no endogenous innovation, this would correspond to the case in which the equilibrium exhibits sustained growth through capital accumulation, or stagnation, with consumption eventually bounded or decreasing. As we shall see, this remains the case with a labor constraint. We take the case of a growing economy, that is $\delta \beta>1$, first.

\subsubsection{The General Case}

We begin by establishing that $\delta \beta>1$ does correspond to sustained growth in per capita consumption and in the quality of capital. First we observe that consumption is non-decreasing:

Lemma 1. Suppose that $\delta \beta>1$. Then $c_{t+1}^{*} \geq c_{t}^{*}$.

Proof. The correspondence $C$ is a stairstep with vertices

$$
\left(\gamma^{i},\left[u^{\prime}\right]^{-1}\left((\beta \delta)^{-t} q_{0}^{0}(\beta / \rho)^{i-1}[(\beta \gamma / \rho)-1] /(\gamma-1)\right)\right) .
$$

Increasing $\delta \beta$ increases the height of the vertex for each $\gamma^{i}$. In addition, the upper bound on the domain of the correspondence, $\gamma^{t}$, grows larger with $t$ as well. It follows that the fixed point $c_{t}^{*}$ must be non-decreasing.

An immediate implication is that the technologies used to produce consumption must be improving over time for, with full employment, consumption would otherwise have to decrease. It is also the case that asymptotically there is no upper bound on the quality of capital used to produce consumption. 
Lemma 2. Suppose that $\delta \beta>1$. Then there is no upper bound on the qualities of capital used to produce consumption.

Proof. Observe that for fixed $i$ as $t \rightarrow \infty$,

$$
\frac{(\beta \delta)^{-t} q_{0}^{0}(\beta / \rho)^{i-1}[(\beta \gamma / \rho)-1]}{(\gamma-1)} \rightarrow 0 .
$$

Hence, for any given $i$, for large enough $t$ the fixed point of $C$ must lie to the right of $\gamma^{i}$, meaning that a better quality capital than $i$ is used to produce consumption (see Figure 1).

For $\delta \beta>1$ the general picture is therefore this. As $t$ grows larger, the correspondence $C$ moves up and to the right. Observe that $C$ has only horizontal and vertical segments. If the correspondence moves upwards sufficiently slowly (which, for given utility function, is more likely the closer $\delta \beta$ is to 1 ) then the fixed point will generally lie on the same horizontal segment for several consecutive periods. This length of time will determine the rate at which new technologies are introduced and the speed of capital widening. The exact mechanics can be appreciated by noticing that the system behaves differently on horizontal and vertical segments. On horizontal segments, two types of capital are used to produce consumption, one of them (the highest in quality) is being accumulated while the other is being phased out. During these periods consumption grows at a rate determined by the speed of accumulation of the highest quality capital, and the correspondence $C$ shifts upwards. We refer to this as a boom. On vertical segments, only one type of capital is used to produce consumption, while a new quality of capital is being introduced by means of the $\rho$ technology. Because the stock of capital used to produce consumption is not increasing, consumption remains constant as the correspondence $C$ shifts upwards with time. We refer to this period as a growth recession. In other words the economy exhibits an endogenous cycle in technological innovation and, therefore, in the growth rate of Total Factor Productivity.

One striking fact is that during a growth recession (which corresponds to periods of technological innovation) the real wage increases together with the share of labor in national income. Specifically, during a recession, consumption is constant, so its present value price declines by a factor $\delta$. On the other hand, the present value price of each quality of capital declines at $1 / \beta<\delta$, and in particular the real price of capital falls. Since only one activity is used to produce consumption, zero profits for this activity implies the real wage must increase. This change in relative prices makes economic sense. During a recession, the real price 
of "higher" quality capital declines, and the real wage increases, until it becomes profitable to introduce the next higher quality of capital in the production of consumption to save on labor. In this sense, technological progress is "biased" in this model as it takes place to conserve a particular factor when its relative price is high enough to make the innovation profitable.

One point to emphasize is the endogeneity of technological progress in this type of equilibrium. Although the fact that the best quality of capital that can be used to produce consumption in period $t$ is exogenously given, it is not this exogenous constraint that determines which technology is actually used in that period. Rather the quality of capital $i$ used to produce consumption in period $t$ is generally lower than the best exogenously available, that is $i<t$. The exact quality of capital used in each period is determined by prices and a profit and loss calculation. Technologies are willingly introduced when it becomes profitable to do so for rational economic agents.

\subsection{The Continuous Time Limit}

We can get a more accurate picture of the cycle by studying a special case. We continue to assume $\delta \beta>1$ and suppose that the effective amount of time $\Delta$ between periods is small. So that the cycle does not depend on time, assume that, at least for consumption exceeding a minimum amount, preferences have the CES form

$$
u\left(c_{t}\right)=-(1 / \theta)\left[c_{t}\right]^{-\theta} .
$$

This assumption, together with the fact that new technologies improve geometrically gives rise when $\Delta$ is small to a cycle length that is essentially independent of time. Let us take $\delta=e^{-r \Delta}, \beta=e^{b \Delta}$, so that the assumption $\delta \beta>1$ corresponds to $b>r$. We also assume that innovations are discrete, so that the extent to which machine $i$ saves on labor relative to machine $i-1$ is independent of the time between periods. Hence $\gamma>1$ independently of $\Delta$. We also have $\rho=\tilde{\rho} e^{d \Delta}$. Since innovations are costly, we assume that $b>d$ and that $\tilde{\rho}<1$. As we are interested only in small values of $\Delta$, we may also assume that $\rho=\tilde{\rho} e^{d \Delta}<\gamma$ for $\Delta$ in the range considered here. We also denote calendar time by $\tau=t \Delta$.

Suppose at some particular time that $c_{t}^{*}=\gamma^{i-1}$. Then

$$
u^{\prime}\left(c_{t}^{*}\right)=(\beta \delta)^{-t} q_{0}^{0}(\beta / \rho)^{i-1}[(\beta \gamma / \rho)-1] /(\gamma-1) .
$$

This corresponds to the beginning of a horizontal segment or a boom. In our 
special case, we may write this as

$$
c_{t}^{*}=\left\{\left(e^{(b-r) \Delta}\right)^{-\tau / \Delta} q_{0}^{0}\left[\left(\frac{e^{(b-d) \Delta}}{\tilde{\rho}}\right)^{i-1} \frac{\gamma e^{(b-d) \Delta}-\tilde{\rho}}{\tilde{\rho}(\gamma-1)}\right]\right\}^{-1 /(\theta+1)} .
$$

As $\tau$ increases, so does $c_{t}^{*}$ until eventually $c_{t}^{*}=\gamma^{i}$, at which point the recession occurs. We can calculate a good approximation to this length of time by taking the continuous time limit when $\Delta \rightarrow 0$

$$
c_{t}^{*}=\left[e^{(r-b) \tau} q_{0}^{0}(1 / \tilde{\rho})^{i-1} \frac{(\gamma / \tilde{\rho})-1}{\gamma-1}\right]^{-1 /(\theta+1)} .
$$

In other words, during the growth period consumption is simply growing at the rate $(b-r) /(\theta+1)$. The length of the boom $\tau_{b}$ is determined by the amount of time required for consumption to grow by a factor of $\gamma$, or

$$
\tau_{b}=\frac{\theta+1}{b-r} \ln \gamma
$$

The recession, on the other hand, lasts from $t$ to $t+\tau_{r} / \Delta$ where

$$
\begin{aligned}
& (\beta \delta)^{-t} q_{0}^{0}(\beta / \rho)^{i-1}[(\beta \gamma / \rho)-1] /(\gamma-1)= \\
& \quad=(\beta \delta)^{\left(t+\tau_{r} / \Delta\right)} q_{0}^{0}(\beta / \rho)^{i}[(\beta \gamma / \rho)-1] /(\gamma-1) .
\end{aligned}
$$

The continuous time approximation gives,

$$
e^{(r-b) \tau_{r}} \frac{e^{(b-d) \Delta}}{\tilde{\rho}}=1
$$

Taking the limit for $\Delta \rightarrow 0$ and solving for $\tau_{r}$

$$
\tau_{r}=-\frac{\ln \widetilde{\rho}}{b-r}
$$

Consider next the length $\tau_{c}=\tau_{b}+\tau_{r}$ of the whole cycle. This is

$$
\tau_{c}=\frac{1}{b-r}\left[\ln \left(\frac{\gamma^{1+\theta}}{\tilde{\rho}}\right)\right]
$$

which is increasing in $\gamma, \theta$ and $r$ and decreasing in $b$ and $\tilde{\rho}$. The shorter the cycle, the more quickly new technologies are introduced, so we find that the frequency 
of innovations respond negatively to the quality of the innovation $\gamma$, the preference parameter $\theta$, and the subjective degree of impatience $r$. The most interesting of these is the quality of the innovation $\gamma$. Higher quality innovations in this model lead to less innovation, because they make it possible to grow for a longer period of time without hitting the labor constraint. On the other hand, we find that there is more innovation if the cost of producing capital, as measured by the inverse of either $b$ or $\tilde{\rho}$, goes down.

The relative length of the two phases, booms and recessions, is

$$
\frac{\tau_{b}}{\tau_{r}}=-(1+\theta) \frac{\ln \gamma}{\ln \tilde{\rho}}
$$

Interestingly enough, neither the productivity of the capital widening technology, nor the degree of impatience affect the relative length of booms and recessions. Economies where people exhibit low willingness to substitute consumption over time (high values of $\theta$ ) have longer (but "less rampant") booms for a given recession length. As we noted above, improved quality of innovation (high $\gamma$ ) makes it possible to grow for a longer period of time without hitting the labor constraint. This increases the length of booms, but not of recessions. Finally, a large cost of innovation is bound to increase the relative amount of time spent in recession.

The average growth rate of consumption over an entire cycle is the value of $g$ solving

$$
\gamma=\exp \left[g \cdot \frac{1}{b-r} \ln \left(\frac{\gamma^{1+\theta}}{\tilde{\rho}}\right)\right] \text {, }
$$

which is

$$
g=\frac{b-r}{1+\theta-\ln \tilde{\rho} / \ln \gamma} .
$$

Hence, economies where people are more willing to substitute consumption over time grow faster on average, as do economies able to implement more substantial innovations.

We already noted above that the real wage grows during recession. In fact, we have somehow argued that our growth recessions are brought about by the increase in the real wage relative to the price of new capital. To save on expensive labor by introducing the relative cheaper new machines, resources must temporarily be shifted to the labor saving innovation, which reduces the growth rate of consumption. As we have constructed our model in such a way that there is always full employment, this implies a countercyclical movement in the labor share of national income. Over the entire cycle, productivity of labor grows of a factor 
$\gamma$, the same for the real wage. As consumption is constant during recessions, its price relative to both old and new capital must be increasing then. Overall, the price of a machine of quality $i$ decreases over time relative to that of consumption and the rate of decrease is uniform across qualities.

\subsection{Stagnation}

Finally we turn to the case in which $\delta \beta \leq 1$. In the absence of a labor constraint, this would imply that the economy remains stagnant, either with constant consumption if $\delta \beta=1$, or declining consumption if $\delta \beta<1$. With the labor constraint, if $\rho>\gamma$ and $\kappa_{0}^{0} \geq \rho /(\rho-\gamma)$ we have already indicated that the equilibrium is the Solow path of exogenous sustained growth regardless of whether $\delta \beta \leq 1$. In this case, introducing a labor constraint and the possibility of factor saving technological progress, changes a stagnant economy in which consumption never grows into an expanding economy in which consumption grows forever, and new technologies are introduced every period. When labor saving innovations are feasible, the addition of a labor constraint can be seen as the incentive toward adopting technological innovations that lead to higher consumption.

On the other hand, the next theorem shows that if either the Solow path does not exist because $\rho<\gamma$, or there is insufficient initial capital, then the picture is indeed one of a stagnant economy in the long run. There is an upper limit on the highest quality of capital ever produced, and ultimately consumption either stops growing $(\delta \beta=1)$ or declines $(\delta \beta<1)$.

Theorem 5. Suppose either $\rho<\gamma$ or $\kappa_{0}^{0}<\rho /(\rho-\gamma)$. If $\delta \beta \leq 1$ there exists a technology I such that no quality of capital greater than I is ever produced, and a period $T$ such that for all $t \geq T$

- If $\delta \beta=1, c_{t}^{*}=c_{T}^{*}$

- If $\delta \beta<1, c_{t+1}^{*}<c_{t}^{*}<1$.

- Only the worst quality capital is used to produce consumption.

Proof. Under the conditions given, it follows that $q_{0}^{0}>0$. As $i \rightarrow \infty$, the Inada condition for $c \rightarrow \infty$ implies 


$$
\begin{aligned}
& {\left[u^{\prime}\right]^{-1}\left((\delta \beta)^{-t} q_{0}^{0}(\beta / \rho)^{i-1}[(\beta \gamma / \rho)-1] /(\gamma-1)\right)} \\
& \leq\left[u^{\prime}\right]^{-1}\left(q_{0}^{0}(\beta / \rho)^{i-1}[(\beta \gamma / \rho)-1] /(\gamma-1)\right) \rightarrow 0 .
\end{aligned}
$$

It follows that there is some technology $I$ for which, for all $t$,

$$
\left[u^{\prime}\right]^{-1}\left((\delta \beta)^{-t} q_{0}^{0}(\beta / \rho)^{I-1}[(\beta \gamma / \rho)-1] /(\gamma-1)\right)<\gamma^{I} .
$$

Consequently, no technology $i \geq I$ is used to produce consumption. It follows that the optimal consumption plan does not ever produce any capital of quality $i \geq I$.

For $\delta \beta=1$ the correspondence $C$ does not either increase or decrease, it simply shifts to the right; once $t \geq I$ it follows that there is a unique and time independent fixed point of $C$. For $\delta \beta<1$, as $t \rightarrow \infty$ we have $\left[u^{\prime}\right]^{-1}\left((\delta \beta)^{-t} q_{0}^{0}\right) \rightarrow 0$, so eventually the fixed point of $C$ must lie below 1 . Since $\left[u^{\prime}\right]^{-1}\left((\delta \beta)^{-t} q_{0}^{0}\right)$ is also strictly decreasing, so is $c_{t}^{*}$. Since the fixed point of $C$ is going to zero, it must be that eventually it falls below 1 , with the implication that only the worst possible technology is used to produce consumption.

This theorem also demonstrates another important possibility in this economy: path dependence. That is, suppose that $\delta \beta<1$ and $\rho>\gamma$. Then if initial capital exceeds the level $\kappa_{0}^{0} \geq \rho /(\rho-\gamma)$ needed for the Solow path, the long run is one of technological innovation and sustained growth. On the other hand, if initial capital falls a bit short of the threshold, so that $\kappa_{0}^{0}<\rho /(\rho-\gamma)$ in the long-run only the lowest possible quality capital is used, there is unemployment, and consumption continually falls. In particular, if we were to compare two economies with different initial capital endowments, one above and one below the threshold, we would discover that they do not "converge" to the same long-run growth path.

Finally, we point out a further interesting property of our economy: consumption and growth paths that, depending upon initial conditions, may be strictly non-monotone. More precisely, even when $\kappa_{0}^{0}<\rho /(\rho-\gamma)$ the economy may innovate and grow for some period of time, before falling back into stagnation. For economies of this kind, relatively rich at the beginning but highly impatient or not very efficient at reproducing already existing capital, consumption will grow at a rate $\gamma>1$ for a while and then decline forever. As the decline is governed by the stairstep correspondence $C$ the decline is uneven until only the worst possible quality of capital is employed at which time (in the CES case) consumption declines geometrically. It is as if the airplane gets off the runway, then falls back 
to the ground. Figure 2 illustrates three different cases, associated with different initial stocks of capital and $\rho>\gamma$. The black monotone upward sloping curve describes the consumption path associated to the Solow case. The combination of the first portion of the Solow consumption path with either one of the downward sloping curves represent different cases of transient growth followed by decline and eventual stagnation. Obviously, in both the latter cases the initial capital has to be lower than $\rho /(\rho-\gamma)$ because, when $\rho>\gamma$, if initial capital is sufficiently high the economy reaches the Solow growth path and remains there.

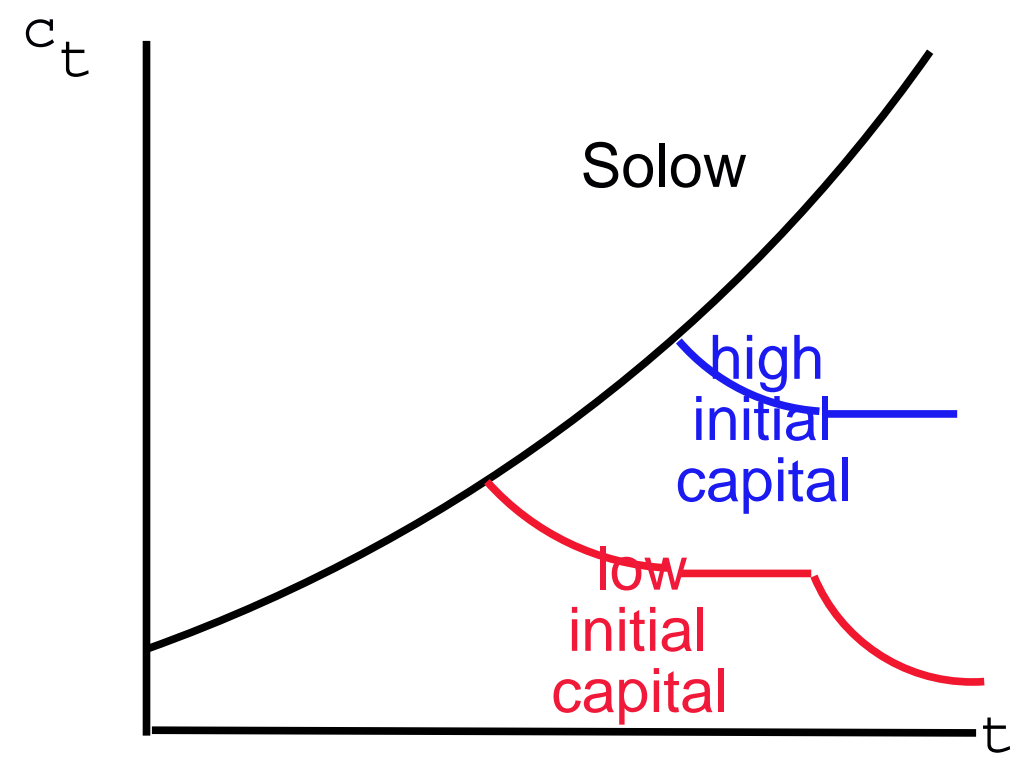

Figure 2 - Stagnation Consumption Paths

\section{Conclusion}

We have examined a model in which an essential input cannot be increased at the same speed as other inputs. Hence, growth in per capita consumption can take place if and only if factor saving innovations are possible. An innovation, new machine, is factor saving when it reduces the input requirement of some factor per unit of output. Machines which, for given output levels, need less inputs than other machines must be more expensive. Hence, factor saving innovations necessarily induce a non-trivial trade-off between capital widening and capital deepening. Capital widening is less costly, but eventually hits a factor constraint (labor in our example) forcing growth through capital deepening. Consequently, 
the rate at which new technologies are introduced becomes endogenous, depending among other things on the rate of intertemporal substitution in consumption, on the technology and on the economy's initial conditions. As in other models of endogenous growth, the rate of growth of consumption is also endogenous in the same sense.

At least since Hicks (1932) seminal writings, economists have been debating about factor saving innovations that are "biased". The latter term indicates that technological progress augments productivity for some factor(s) more than for others and it does so because of relative price incentives. We have built a general equilibrium model capturing this intuition and looked at its implications. Our main finding is that, in these circumstances, technological adoption is likely to be endogenous and, indeed, affected by relative prices and initial conditions. Further, we have proved that when technological progress is factor saving it must come in cycles unless very special circumstances occur.

We have chosen to model the factor constraint as binding in the consumption sector only, and to concentrate on the case of just one fixed factor. However, the basic message remains the same regardless of such simplifying restrictions. Obviously, when the scarce factor can grow at a rate $n$, the whole analysis can be replicated for $\beta>1+n$. When more than one scarce factor exists, factor saving innovations can take place along different directions. While this may complicate the model and its equilibrium dynamics, providing an interesting topic of future extensions, the basic message about the oscillatory nature of factor saving technological innovation would only be strengthened. Finally, the basic message does not depend upon the sector in which the constraint binds, or whether labor is perfectly mobile between the two sectors. Indeed, we worked out preliminary versions of the case of perfect labor mobility between the two sectors, without qualitatively different results. Notice, incidentally, that our example does not require that there be no labor used in the production of capital, just that there is labor immobility between the two sectors, and that the labor constraint binds first in the consumption sector. This particular example is a useful starting point because of its simplicity and the stark results it delivers. In addition we do not think the assumption of perfect labor mobility between the two sectors is especially more plausible than complete immobility between the two sectors. 


\section{Appendix}

Lemma A. A consumption plan $c$, with $c_{t}>0$ for all $t$, maximizes $U(c)$ subject to the budget constraint if and only if for some $\psi \geq 0$

$$
\begin{aligned}
p_{t} & =\psi \delta^{t} u^{\prime}\left(c_{t}\right) \\
\sum_{t=0}^{\infty} p_{t} c_{t} & =q_{0}^{0} \kappa_{0}^{0}+q_{0}^{\ell}
\end{aligned}
$$

Proof. This is standard.

Theorem 1. Suppose that $\lambda^{*}, c^{*}$ is a feasible allocation for the initial condition $z_{0}$. Then $\lambda^{*}, c^{*}$ solves the social planner problem if and only if we can find prices $q, p$ such that $q, p, \lambda^{*}, c^{*}$ are a competitive equilibrium..

Proof. That a competitive equilibrium solves the social planner problem is a standard first welfare theorem proof. To prove the second, we need to show that we can find prices that support a solution to the social planner problem.

Suppose that $\lambda, c^{*}$ is a solution to the planner problem for the initial condition $z_{0}$. Let $z^{*}$ be the corresponding inputs. Let $z_{T+1}$ denote a vector of labor and capital of quality $i \leq T+1$. Let $V^{T+1}\left(z_{T+1}\right)$ denote the maximum utility, discounted at $t=0$, of beginning with the endowment $z_{T+1}$ in period $T+1$ and continuing forward. Let $U^{T}(c)=\sum_{t=0}^{T} \delta^{t} u\left(c_{t}\right)$. Observe that $\lambda^{*}, c^{*}$ solves the problems of maximizing $U^{T}(c)+V^{T+1}\left(z_{T+1}\right)$ subject to social feasibility. This is a finite dimensional problem. By standard finite dimensional arguments, we can find finite dimensional price vectors $\bar{q}^{T}, \bar{p}^{T}$ so that the zero profit conditions are satisfied for viable activities up to $T+1$. By the same standard arguments, the vector $c_{t}^{*}, t=0, \ldots, T$ and the scalar $z_{T+1}^{*}$ are optimal for the consumer under the budget constraint $\sum_{t=0}^{T} \bar{p}_{t}^{T} c_{t}+\bar{q}_{T+1}^{T} z_{T+1}=\bar{q}_{0}^{\kappa T} \kappa_{0}^{0}+\bar{q}_{0}^{\ell T}$.

Now we may normalize prices so that $\bar{p}_{t}=\delta^{t} u^{\prime}\left(c_{t}^{*}\right)$. Let $Q^{T}$ denote the set of all (non-negative) infinite dimensional price sequences for which the projection on $\mathfrak{R}_{+}^{T} \times \mathfrak{R}_{+}^{2 \times(T+1)}$ is a supporting price vector for the finite dimensional problem above. Observe that $Q^{T} \supseteq Q^{T+1}$ and that these are closed spaces. It follows that $Q=\cap_{T \rightarrow \infty} Q^{T}$ is closed, although possibly empty.

Next observe that $\bar{q}_{t+1}^{T}$ is a supergradient of $V^{t+1}\left(z_{t+1}\right)$ at $z_{t+1}^{*}$. Notice that $\bar{q}_{t+1}^{T}$ is bounded below by zero and above by some finite two dimensional vector as $\bar{q}_{t+1}^{T} z_{t+1}^{*}+V^{t+1}(0) \leq V^{t+1}\left(z_{t+1}^{*}\right)$ and the latter is finite. It follows that the intersection $Q$ is nonempty. 
Let $q$ be in $Q$. By construction $q$ and $p$ (which is uniquely defined from the first order condition) satisfy zero profits. From the consumer budget constraint in the truncated problems, we have $\sum_{t=0}^{T} p_{t} c_{t}^{*}+q_{T+1} z_{T+1}^{*}=q_{0}^{\kappa_{1}} \kappa_{0}^{0}+q_{0}^{\ell}$. Since $\bar{q}_{t+1}$ is a gradient of $V^{t+1}\left(z_{t+1}\right)$ at $z_{t+1}^{*}$, we have $q_{T+1} z_{T+1}^{*}+V^{T+1}(0) \leq V^{T+1}\left(z_{t+1}^{*}\right)$. Also $V^{T+1}(0)=\delta^{T+1} u(0) /(1-\delta)$ and $V^{T+1}\left(z_{T+1}^{*}\right)=\sum_{t=T+2}^{\infty} \delta^{t} u\left(c_{t}^{*}\right)$. Since $\sum_{t=0}^{\infty} \delta^{t} u\left(c_{t}^{*}\right)<\infty$ it follows that $\lim _{T \rightarrow \infty} V^{T+1}\left(z_{T+1}^{*}\right) \rightarrow 0$, and so $q_{T+1} z_{T+1}^{*} \rightarrow 0$, which gives $\sum_{t=0}^{\infty} p_{t} c_{t}^{*}=q_{0}^{0} \kappa_{0}^{0}+q_{0}^{\ell}$

Theorem 2. For given $z_{0}$, a competitive equilibrium exists, and there is unique competitive equilibrium consumption plan $c^{*}$

Proof. Since $U(c)$ is bounded above on the feasible set of feasible consumption paths, it is continuous in the product topology. Since this set is compact in the product topology, an optimum exists; it is unique since $U$ is strictly concave.

Define a simple plan to be a pair of sequences of integers $(v, \eta)=\left(v_{0}, \eta_{0}, v_{1}\right.$, $\left.\eta_{1}, \ldots\right)$ where $v_{t} \in\{1,2\}, v_{0}=1 ; t \geq \eta_{t} \geq 0$, and $\eta_{t}>0$ if $v_{t}=2$. A production plan $(\lambda, k)$ is consistent with the simple plan $(\nu, \eta)$ if

1. exactly $v_{t}$ different qualities of capital are employed in period $t$ to produce consumption

2. when $v_{t}=1$ the quality of capital employed to produce consumption is $\eta_{t}$, and

3. when $v_{t}=2$ the two qualities of capital used to produce consumption are $\eta_{t}, \eta_{t}-1$.

We say that a production plan exhibits full employment if there is unemployment only in periods where no quality of capital other than 0 is used to produce consumption. We say that a simple plan $(v, \eta)$ and a consumption stream $c$ are consistent if there is a full employment production plan $(\lambda, k)$ consistent with the simple plan that yields the output $c$. If $\mathrm{v}_{t}=1$ and $\eta_{t}=0$ then $(\lambda, k)$ uses exactly $\kappa_{t}^{\eta_{t}}=c_{t} \leq 1$ units of quality 0 capital. If $v_{t}=1$ and $\eta_{t}>0$ then it uses $\kappa_{t}^{\eta_{t}}=\gamma^{\eta_{t}}$ units of quality $\eta_{t}$ capital for full employment, so $c_{t}=\gamma^{\eta_{t}}$. If $v_{t}=2$ then we it uses exactly $\kappa_{t}^{\eta_{t}}$ units of quality $\eta_{t}$ capital and exactly $\kappa_{t}^{\eta_{t}-1}$ units of quality $\eta_{t}-1$ capital, where these are the unique solution of $\kappa_{t}^{\eta_{t}} / \gamma^{\eta_{t}}+\kappa_{t}^{\eta_{t}-1} / \gamma^{\eta_{t}-1}=1$, and $\kappa_{t}^{\eta_{t}}+\kappa_{t}^{\eta_{t}-1}=c_{t}$. For convenience, we now replicate the definitions from the text. 
For any given value of $c_{t}$ observe that either $c_{t} \leq 1$ or for some $i>0, \gamma^{i-1}<c_{t} \leq \gamma^{i}$. In the former case define $\eta\left(c_{t}\right)=0$, in the latter $\eta\left(c_{t}\right)=i$. Let

$$
\kappa_{0}^{0}\left(c_{t}\right)=\left\{\begin{array}{cc}
\beta^{-t} c_{t} & \eta\left(c_{t}\right)=0 \\
\beta^{-t}\left(\frac{\beta}{\rho}\right)^{\eta\left(c_{t}\right)} \frac{\gamma c_{t}-\gamma^{\eta\left(c_{t}\right)}}{\gamma-1}+\beta^{-t}\left(\frac{\beta}{\rho}\right)^{\eta\left(c_{t}\right)-1} \frac{\gamma^{\eta\left(c_{t}\right)}-c_{t}}{\gamma-1} & \eta\left(c_{t}\right)>0
\end{array}\right.
$$

and

$$
\kappa_{0}^{0}(c)=\sum_{t=0}^{\infty} \kappa_{0}^{0}\left(c_{t}\right)
$$

Set

$$
\begin{aligned}
& \zeta_{0}=1 \\
& \zeta_{i}=(\beta / \rho)^{i-1}[(\beta \gamma / \rho)-1] /(\gamma-1) .
\end{aligned}
$$

Define the correspondence $c_{t}^{\prime} \in C_{t}\left(c_{t}, q_{0}^{0}\right)$ by

$$
\begin{array}{ll}
u^{\prime}\left(c_{t}^{\prime}\right)=(\beta \delta)^{-t} q_{0}^{0} \zeta_{\eta\left(c_{t}\right)} & \text { if } c_{t}<\gamma^{\eta\left(c_{t}\right)}, \eta\left(c_{t}\right) \leq t \\
(\beta \delta)^{-t} q_{0}^{0} \zeta_{\eta\left(c_{t}\right)} \leq u^{\prime}\left(c_{t}^{\prime}\right) \leq(\beta \delta)^{-t} q_{0}^{0} \zeta_{\eta\left(c_{t}\right)+1} & \text { if } c_{t}=\gamma^{\eta\left(c_{t}\right)}, \eta\left(c_{t}\right)<t \\
(\beta \delta)^{-t} q_{0}^{0} \zeta_{\eta\left(c_{t}\right)} \leq u^{\prime}\left(c_{t}^{\prime}\right) & \text { if } c_{t}=\gamma^{\eta\left(c_{t}\right)}, \eta\left(c_{t}\right)=t
\end{array}
$$

This correspondence is upper-hemi-continuous, convex valued and non-increasing.

Theorem 3. For given $z_{0}$ the feasible consumption plan $c^{*}$ is an optimum if and only if there exists a $q_{0}^{0}$ such that

$$
\kappa_{0}^{0} \geq \kappa_{0}^{0}\left(c^{*}\right) \text { with equality unless } q_{0}^{0}=0,
$$

and

$$
c_{t}^{*} \in C_{t}\left(c_{t}^{*}, q_{0}^{0}\right)
$$

Moreover, equilibrium prices are given by

$$
\begin{gathered}
q_{t}^{i}=\beta^{-t}\left(\frac{\beta}{\rho}\right)^{i} q_{0}^{0} \\
w_{t}=\gamma^{\eta\left(c_{t}^{*}\right)}\left[\delta^{t} u^{\prime}\left(c_{t}^{*}\right)-\beta^{t}(\beta / \rho)^{\eta_{t}} q_{0}^{0}\right]
\end{gathered}
$$

and

$$
q_{T}^{\ell}=\sum_{t=T}^{\infty} w_{t}
$$

The equilibrium production plan is any feasible plan that produces $c_{t}^{*}$ using only quality $\eta\left(c_{t}^{*}\right)$ and $\eta\left(c_{t}^{*}\right)-1$ quality capital, and has full employment whenever $\eta\left(c_{t}^{*}\right)>0$. 
Proof. For given $z_{0}$ suppose the feasible consumption plan $c^{*}$ is an optimum. We first claim that there is an initial price of capital $q_{0}^{0}$, a non-negative sequence of wages $w=\left(w_{0}, w_{1}, \ldots\right)$ and a simple plan $(v, \eta)$ consistent with $c^{*}$ such that the following conditions hold

1) $\delta^{t} u^{\prime}\left(c_{t}^{*}\right)-\beta^{-t}(\beta / \rho)^{\eta_{t}} q_{0}^{0}-w_{t} / \gamma^{\eta_{t}}=0$;

2) if $v_{t}=2$ then $\delta^{t} u^{\prime}\left(c_{t}^{*}\right)-\beta^{-t}(\beta / \rho)^{\eta_{t}-1} q_{0}^{0}-w_{t} / \gamma^{\eta_{t}-1}=0$;

3) if $v_{t}=1$ and $\eta_{t}<t$ then $\delta^{t} u^{\prime}\left(c_{t}^{*}\right)-\beta^{-t}(\beta / \rho)^{\eta_{t}+1} q_{0}^{0}-w_{t} / \gamma^{\eta_{t}+1} \leq 0$;

4) if $v_{t}=1$ and $\eta_{t}>0$ then $\delta^{t} u^{\prime}\left(c_{t}^{*}\right)-\beta^{-t}(\beta / \rho)^{\eta_{t}-1} q_{0}^{0}-w_{t} / \gamma^{\eta_{t}-1} \leq 0$;

5) $w_{t}=0$ if there is unemployment at $t$.

First observe that if capital of quality $i>0$ is used to produce consumption for period $t$ and there is unemployment, strictly greater consumption in that period can be had by replacing quality $i$ capital in period $t$ with quality 0 . The full employment condition is consequently necessary for an optimum along any path that uses capital of quality other than $i=0$.

We now apply the zero profit conditions for competitive equilibrium. Let $q_{t}^{i}$ denote the price of quality $i$ capital in period $t$, and let $q_{t}^{\ell}$ denote the price of labor. Beginning with one unit of quality 0 capital in period $0, i$ quality upgrades producing $\rho$ units of capital each and $t-i$ periods producing $\beta$ units of capital are required to get from quality 0 at time 0 to quality $i$ at time $t$, for all possible $i \leq t$. The order in which the $\rho$ and $\beta$ phases come, does not matter. It follows then, from the zero profit condition applied to the capital producing activities only, that if $i \leq t$ then

$$
q_{t}^{i}=\frac{q_{0}^{0}}{\rho^{i} \beta^{t-i}}=\beta^{-t}\left(\frac{\beta}{\rho}\right)^{i} q_{0}^{0} .
$$

From the fact that labor can always reproduce itself, we have

$$
q_{t}^{\ell} \geq q_{t+1}^{\ell}
$$

with equality if there is unemployment in period $t$. So we may define the wage rate

$$
w_{t}=q_{t}^{\ell}-q_{t+1}^{\ell} \geq 0 .
$$

We may then write the profits from the activity that at time $t$ produces consumption $c_{t+1}$ from quality $i$ capital as

$$
\pi_{t}^{i}=\delta^{t} u^{\prime}\left(c_{t}\right)-\beta^{-t}(\beta / \rho)^{i} q_{0}^{0}-w_{t} / \gamma^{i} .
$$

Recall that in equilibrium profits must be non-positive. Observe that this function is strictly concave as a function of $i$ for fixed values of $c_{t}, q_{0}^{0}, w_{t}$. It follows that 
if this is non-positive for all $i \leq t$ it is zero for at most two activities, in which case is strictly negative for all other activities, or if it is zero for one activity, it is sufficient that it be non-positive for the next highest and next lowest ones to be non-positive for all activities. So since in equilibrium $p_{t}=\delta^{t} u^{\prime}\left(c_{t}^{*}\right)$ conditions 1)-5) are indeed necessary.

Next we observe that $c_{t}^{*} \in C_{t}\left(c_{t}^{*}, q_{0}^{0}\right)$ if and only if (1)-(4) hold. In case $c_{t}<$ $\gamma^{\eta\left(c_{t}\right)}$ full employment requires that $c_{t}$ be produced using qualities $\gamma^{\eta}\left(c_{t}\right), \gamma^{\eta}\left(c_{t}\right)-1$ of capital. Then $v_{t}=2$ and (1) and (2) must hold. Solving yields $u^{\prime}\left(c_{t}^{*}\right)=$ $(\beta \delta)^{-t} q_{0}^{0} \zeta_{\eta\left(c_{t}^{*}\right)}$ and

$$
w_{t}=\frac{\beta^{-t}\left[(\beta / \rho)^{\eta\left(c_{t}^{*}\right)}-(\beta / \rho)^{\eta\left(c_{t}^{*}\right)-1}\right] q_{0}^{0}}{1 / \gamma^{\eta\left(c_{t}^{*}\right)-1}-1 / \gamma^{\eta\left(c_{t}^{*}\right)}}
$$

which is non-negative since $\beta / \rho \geq 1$ and $1 / \gamma<1$.

Turning to $c_{t}=\gamma^{\eta}\left(c_{t}\right)$, we have (1), (3) and (4)

$$
\begin{array}{r}
\delta^{t} u^{\prime}\left(c_{t}^{*}\right)-\beta^{-t}(\beta / \rho)^{\eta\left(c_{t}^{*}\right)} q_{0}^{0}-w_{t} / \gamma^{\eta\left(c_{t}^{*}\right)}=0 \\
\delta^{t} u^{\prime}\left(c_{t}^{*}\right)-\beta^{-t}(\beta / \rho)^{\eta\left(c_{t}^{*}\right)+1} q_{0}^{0}-w_{t} / \gamma^{\eta\left(c_{t}^{*}\right)+1} \leq 0 \\
\delta^{t} u^{\prime}\left(c_{t}^{*}\right)-\beta^{-t}(\beta / \rho)^{\eta\left(c_{t}^{*}\right)-1} q_{0}^{0}-w_{t} / \gamma^{\eta\left(c_{t}^{*}\right)-1} \leq 0
\end{array}
$$

We can solve the first equation for $w_{t}$. Substituting into the second inequality, we see that it is satisfied if any only if $u^{\prime}\left(c^{*}\right) \leq(\beta \delta)^{-t} q_{0}^{0} \zeta_{\eta\left(c_{t}\right)}$, and the first if and only if $(\beta \delta)^{-t} q_{0}^{0} \zeta_{\eta\left(c_{t}\right)-1} \leq u^{\prime}\left(c^{*}\right)$. It is easy to check that these two inequalities also imply that $w_{t} \geq 0$.

Finally observe that $\kappa_{0}^{0} \geq \kappa_{0}^{0}\left(c^{*}\right)$ with equality unless $q_{0}^{0}=0$ since otherwise it would be possible to produce $c^{*}$ with less than the initial capital stock.

This proves that the conditions of the Theorem are necessary for an equilibrium. To show that they are also sufficient, observe that the Inada conditions imply that $u^{\prime}(\cdot)$ maps $[0, \infty)$ onto itself, hence, for every $q_{0}^{0}>0$, there is a $\kappa_{0}^{0}$ for which $q_{0}^{0}$ is the equilibrium price of capital. Let $c^{*}$ be the corresponding optimal consumption. This satisfies the necessary condition $c_{t}^{*} \in C_{t}\left(c_{t}^{*}, q_{0}^{0}\right)$ and $\kappa_{0}^{0}=\kappa_{0}^{0}\left(c^{*}\right)$. Since $c_{t}^{*} \in C_{t}\left(c_{t}^{*}, q_{0}^{0}\right)$ has a unique solution, it follows that these conditions are sufficient as well.

\section{References}

Boldrin, M. and D.K.Levine (2000), "Perfectly Competitive Innovation”, mimeo, University of Minnesota and UCLA. 
Hicks, J.R. (1932), The Theory of Wages, London: MacMillan, 1963, (First edition, 1932).

Jones, L. E. and R. Manuelli (1990), “A Convex Model of Equilibrium Growth: Theory and Policy Implications", Journal of Political Economy, 98, 1008-1038.

Lucas, R.E. Jr. (1988), "On the Mechanics of Economic Development”, Journal of Monetary Economics, 24, 1-55.

Prescott, E. (1998), "Needed: A Theory of Total Factor Productivity," International Economic Review, 39, 525-551

Rebelo, S. (1991), "Long Run Policy Analysis and Long Run Growth,” Journal of Political Economy, 99, 500-521.

Romer, P. (1990), “Endogenous Technological Change," Journal of Political Economy, 98 , S-S.

Romer, P. (1994), “The Origins of Endogenous Growth,"Journal of Economic Perspectives, 8, 3-22. 